\title{
Primary manual aspiration thrombectomy (MAT) for acute ischemic stroke: safety, feasibility and outcomes in 112 consecutive patients
}

\author{
Brian Jankowitz, ${ }^{1}$ Ramesh Grandhi, ${ }^{1}$ Anat Horev ${ }^{2}$ Amin Aghaebrahim, ${ }^{2}$ \\ Ashutosh Jadhav, ${ }^{2}$ Guillermo Linares, ${ }^{2}$ Tudor Jovin ${ }^{2}$
}

${ }^{1}$ Department of Neurosurgery, University of Pittsburgh Medical Center, Pittsburgh, Pennsylvania, USA

${ }^{2}$ Department of Neurology, Stroke Institute, University of Pittsburgh Medical Center, Pittsburgh, Pennsylvania, USA

\section{Correspondence to} Dr Tudor Jovin, Department of Neurology, UPMC Stroke Institute, University of Pittsburgh Medical Center, 200 Lothrop Street, PUH, C-400, Pittsburgh, PA 15213, USA jovitg@upmc.edu

Received 27 October 2013 Accepted 4 December 2013 Published Online First 2 January 2014

\section{CrossMark}

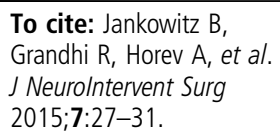

\section{ABSTRACT}

Aim To describe procedural aspects and clinical outcomes in a consecutive series of patients in whom manual aspiration thrombectomy (MAT) was performed as the first treatment modality with other techniques used only in case MAT did not yield recanalization. Methods A retrospective review of a prospectively acquired acute stroke intervention database was performed. Primary MAT was carried out with a preference for the largest catheter considered to be trackable into the target occlusive lesion. The catheter was wedged into the thrombus followed by manual aspiration with a $20 \mathrm{ml}$ syringe.

Results 112 consecutive patients were evaluated. The median age was 66 years and the median NIH Stroke Scale score was 17. Occlusion locations included the M1 (62\%), M2 (8\%), internal carotid artery terminus (19\%) and the vertebrobasilar artery (11\%). Patients with anterior occlusions had tandem extracranial/intracranial occlusive lesions in $18.7 \%$ Median time from symptom onset to groin puncture was $267 \mathrm{~min}$, and from groin puncture to recanalization was $70 \mathrm{~min}$. Successful recanalization (defined as Thrombolysis in Cerebral Infarction (TICl) 2b/3) with primary MAT was $59 \%$ with a median of two passes. $41 \%$ of patients required the use of adjunctive therapy yielding an overall recanalization rate of $\mathrm{TICl} 2 \mathrm{~b} / 3(86 \%)$ and $\mathrm{TICl} 3$ (30.6\%). Parenchymal hematoma of any type ( $\mathrm{PH} 1 / \mathrm{PH} 2)$ was seen in $9.8 \%$ of patients, with symptomatic hemorrhage in $6 \%$. Favorable outcomes (90-day modified Rankin Scale $\leq 2$ ) were $46 \%$. Mortality at 3 months was $31 \%$. Primary MAT was associated with faster procedural times (mean 63 vs 97 min, $p<0.0001$ ) but not with higher rates of favorable outcomes.

Conclusions Primary MAT is an alternative endovascular recanalization technique with reasonable first pass efficacy that will likely improve with technology and experience.

\section{INTRODUCTION}

For intracranial large vessel occlusive disease, recanalization within the fastest possible time frame predicts improved outcomes. ${ }^{1}$ Intravenous tissue plasminogen activator (tPA), the only FDA-approved treatment for acute stroke, is associated with low recanalization rates for large vessel occlusions. $^{2}$ Alternative methods of opening blocked vessels include intra-arterial tPA and an assortment of mechanical devices. Randomized trials in the field of interventional cardiology have shown that manual catheter aspiration is clinically equivalent to other mechanical thrombectomy devices, shortens procedure times, reduces complication rates and is cost-saving. ${ }^{3}{ }^{4}$ Several reports in the literature have used similar techniques in acute ischemic stroke (AIS) interventions, described variously as manual aspiration thrombectomy (MAT), the ADAPT technique, forced suction thrombectomy or thromboaspiration. ${ }^{5-8}$ We have previously described our aspiration technique in 191 patients with acute stroke. ${ }^{6}$ The use of the Merci retriever (Concentric Medical, Mountain View, California, USA) in $89 \%$ of patients, primarily to facilitate advancing the aspiration catheter through the tortuous intracranial vasculature, obscured the potential utility or benefit of aspiration alone. Encouraged by improvements to this technique and the advent of new aspiration catheters, we decided to perform primary MAT in all acute stroke interventions when enrollment in clinical trials did not mandate a different treatment. The purpose of this study is to describe the technique, safety and efficacy of attempting to apply primary MAT upfront for removing intracranial thrombi in a single-center consecutive case series.

\section{METHODS}

\section{Patient selection}

We performed a retrospective analysis of a prospectively acquired database of all patients treated with endovascular therapy for stroke from September 2012 to June 2013 at our institution. Patients receiving intra-arterial stroke treatment at the University of Pittsburgh Medical Center are included in an Institutional Review Board approved prospective database after written informed consent is obtained by the patient or proxies. All patient information was de-identified and analyzed in compliance with Health Insurance Portability and Accountability Act regulations.

All patients presenting with AIS symptoms underwent a baseline head CT scan. In general, as a first step, patients with anterior circulation stroke were selected for intra-arterial therapy if CT showed an Alberta Stroke Program Early CT score of $>6$ or less than one-third hypodensity of the middle cerebral artery (MCA) territory. Most patients underwent additional imaging studies (CT angiography/CT perfusion) or $\mathrm{MRI} / \mathrm{MR}$ angiography and were considered for interventional therapy based on assessment of mismatch between 
the extent of infarcted brain relative to the extent of threatened but viable brain. In patients undergoing CT/CT perfusion, the ratio of low cerebral blood volume (threshold for infarct tissue $\leq 2.0 \mathrm{~mL} \times 100 \mathrm{~mL}$ ) to elevated mean transit time maps and/or to the severity of clinical deficit (NIH Stroke Scale (NIHSS)) was factored into the treatment decision. The minimum amount of mismatch necessary for treatment selection varied according to patient-specific considerations and stroke neurologist/ interventionalist-specific practice patterns. In general, patients with a core volume less than one-third of the MCA territory in the presence of $\mathrm{M} 1$ occlusion and corresponding clinical deficit (NIHSS score $>6-8$ ) or severe perfusion deficit (time to peak $>6 \mathrm{~s}$ ) involving two-thirds or more of the MCA territory were considered potential treatment candidates. Computer-generated volumetric analysis was not available and manual calculation of volumes is too lengthy a process to be useful for consideration in acute stroke intervention. Volumes were therefore estimated based on visual mismatch. Time from stroke onset was not considered a limiting factor. All patients who underwent intra-arterial treatment had a baseline modified Rankin Scale (mRS) score of $\leq 1$.

\section{Treatment algorithm}

After obtaining informed consent, patients underwent catheterbased angiography. Conscious sedation was used in preference to general anesthesia. A micropuncture kit was used to advance a $6 \mathrm{~F}$ sheath into the common femoral artery. Heparin was not routinely used. Once the occlusion was verified, V-18 control wires were used to exchange the diagnostic catheter for either a $6 \mathrm{~F}$ Cook shuttle sheath (Shuttle-SL; Cook Medical, Bloomington, Indiana, USA) or a Neuron MAX 088 (Penumbra, Alameda, California, USA). These base catheters were advanced as distal as possible into the target vessel over their co-packaged tapered dilators. Balloon guide catheters were never used. In the internal carotid artery (ICA), the Cook shuttle sheath was typically advanced to the skull base while the Neuron MAX was often advanced up into the cavernous segment. In the posterior circulation the same base catheters were advanced into the distal V2 segment. If there was a tandem lesion in the extracranial carotid, a stent was typically placed followed by advancement of the base catheter through the stent. These patients were initially managed with intravenous heparin and aspirin followed by loading with $300 \mathrm{mg}$ clopidogrel if no hemorrhagic transformation was visualized after $24 \mathrm{~h}$. Under roadmap guidance, a triaxial system consisting of an aspiration catheter, a microcatheter and guidewire were advanced as distal as possible. We initially used a Merci 18L microcatheter (Concentric Medical, Mountain View, California, USA) and a Synchro 0.014 inch wire (Stryker Neurovascular, Freemont, California, USA), but changed to using a larger Excelsior XT-27 (Stryker Neurovascular) or Marksman (Covidien, Irvine, California, USA) microcatheter with a Fathom 0.016 inch wire (Stryker Neurovascular). When choosing an aspiration catheter, we favored the largest available distal internal diameter in order to maximize the aspiration force. The guidewire and microcatheter were advanced past the clot and into the distal M2 or P2 segment, which provided the necessary support to track the aspiration catheter. Microcatheter injections distal to the thrombus were not part of a standard protocol as this practice was left to the discretion of the operator. If the catheter could not be advanced into the thrombus, it was considered a failed attempt and was counted as an attempted pass. The most common reason for this to occur was the inability to track past the ophthalmic artery, either due to the ledge effect or excessive friction from tortuosity. Typically, a second smaller aspiration catheter was then used such as the 4MAX (Penumbra) or 044 DAC (Stryker Neurovascular). Every attempt was made to wedge the aspiration catheter as distal as possible into the clot. Subsequently, the microcatheter and wire were removed and a $20 \mathrm{ml}$ syringe was attached directly to the hub of the aspiration catheter. Continuous manual aspiration was performed as the catheter was slowly withdrawn through the base catheter. Once the aspiration catheter was removed, the base catheter was also aspirated twice with a $20 \mathrm{ml}$ syringe directly from the hub after removing the hemostatic valve. The choice of aspiration catheter and number of attempts was left up to the primary operator. If residual thrombus was seen in the M2 or P1 segment, a smaller aspiration catheter was used and counted as a successive pass. Typically, if no angiographic improvement was noted or if no thrombus was retrieved through the syringe after two passes, an adjunctive device was used. These devices included the Merci retriever, Solitaire or Trevo; t-PA was not routinely used.

Recanalization (Thrombolysis in Myocardial Infarction (TIMI) and Thrombolysis in Cerebral Infarction (TICI) scores) was recorded by the treating interventionalist and was deemed successful if post-procedure angiography revealed TICI $\geq 2 b$ flow. 9 ${ }^{10}$ The 90-day mRS score was determined at the patient's follow-up appointment. If patients were physically unable to attend these appointments, they or their next of kin were reached by $\mathrm{mRS}$-certified stroke nurse practitioners or stroke fellows and outcomes were obtained over the telephone. Good outcomes were defined as $\mathrm{mRS} \leq 2$. Embolization to a previously uninvolved or new territory (ENT) was defined as any new vessel occlusion or infarct in a previously unaffected territory.

\section{Statistical analysis}

Statistical analysis was performed using STATA IC V.10 software (StataCorp LP, College Station, Texas, USA). Descriptive statistics were performed. In univariate analysis, several variables of interest were correlated with a good functional outcome. For each endpoint, all covariates with $\mathrm{p}<0.2$ were then entered into a multivariate stepwise logistic regression model; significant association was considered for $\mathrm{p}<0.05$.

\section{RESULTS}

The study included 112 consecutive patients of median age 66 years and median NIHSS score 17 . Occlusion locations were as follows: M1 (62\%), M2 (8\%), ICA terminus (19\%), vertebrobasilar artery (11\%). Tandem lesions were encountered in $18 \%$ of patients. Median times from symptom onset to groin puncture and from groin puncture to recanalization were 267 and $70 \mathrm{~min}$, respectively. The range from symptom onset to groin puncture was 70-2058 min. Our series also included 12 wake-up strokes. Final recanalization rates were TICI $2 b / 3$ $(86 \%)$ and TICI 3 (30\%). Primary MAT achieved successful recanalization with a single aspiration catheter in 46 of 112 patients (41\%); another catheter was necessary in 20 patients. Thus, primary MAT was successful in 66 of 112 patients (59\%) when using one or more aspiration catheters. Aspiration was carried out with the following catheter makes and sizes (in inches): Navien (Covidien) 0.072 and 0.058 (84\% of cases), Penumbra (Penumbra) 0.054 and 0.041 (23\% of cases) and DAC (Stryker) $0.070,0.054$ and 0.44 (4\% of cases). Largest bore catheters $(\geq 0.070$ inch) were used in $27 \%$ of cases and medium size catheters $(0.054-0.058$ inch) in 73\%. Neither catheter make nor size was associated with higher or faster recanalization rates. An adjunctive device was used in 46 of 112 patients (41\%), consisting of a stent retriever in $32(28.5 \%)$, a 
Merci device in six (5.3\%), intra-arterial tPA in three $(2.6 \%)$ or a combination of multiple modalities in five (4.4\%). Of the 21 tandem lesions, 11 underwent extracranial stent placement prior to addressing the intracranial occlusion.

Good outcomes occurred in 52 of the 112 patients (46\%) and there were 35 deaths (31\%). Four (3.5\%) intracranial distal wire perforations led to cessation of the procedure. Three perforations resulted in symptomatic intracerebral hemorrhage and clinical deterioration. ENT was documented in four of 112 patients $(3.5 \%)$, with two of 66 events in the primary MAT group and two of 46 events in the adjunctive device group. There were no intracranial dissections but there were four extracranial ICA dissections presumably related to placement of the shuttle sheath or Neuron MAX catheter into the distal ICA.
One type II dissection was treated with intravenous heparin and angioplasty, one type II dissection was stented and two type I dissections were treated with aspirin. No significant intracranial vasospasm was caused by the aspiration catheters. The baseline clinical variables, treatment modalities and predictors of good outcome are shown in table 1.

\section{DISCUSSION}

To our knowledge, this report is the first consecutive case series describing the feasibility, safety and clinical results of primary manual aspiration for AIS. Previous reports including our own published experience have described this approach either as adjunctive therapy to other mechanical embolectomy modalities or in non-consecutive case series. Our preliminary results

Table 1 Baseline clinical variables, treatment modalities and univariate analysis for predictors of good outcome (mRS $\leq 2)$ at 90 days

\begin{tabular}{|c|c|c|c|c|c|}
\hline \multirow[b]{2}{*}{ Variable } & \multirow[b]{2}{*}{$\mathrm{N}(\%)$} & \multirow[b]{2}{*}{ Median (IQR) } & \multicolumn{3}{|c|}{$\mathrm{mRS} \leq 2^{*}$} \\
\hline & & & OR & $p$ Value & $95 \% \mathrm{Cl}$ \\
\hline Age (years) & & $66(29-93)$ & 0.97 & 0.041 & 0.94 to 0.99 \\
\hline NIHSS score & & $17(4-36)$ & 0.78 & 0.000 & 0.70 to 0.88 \\
\hline Baseline ASPECT score & & $9(4-10)$ & 1.50 & 0.008 & 1.11 to 2.04 \\
\hline Time to procedure $(h)^{*}$ & & $4.45(1.16-34.3)$ & 0.99 & 0.113 & 0.99 to 1.00 \\
\hline Treatment time $(\mathrm{min}) \dagger$ & & $70(15-289)$ & 0.99 & 0.047 & 0.980 to 0.99 \\
\hline Men & $61(54.4)$ & & 1.63 & 0.215 & 0.75 to 3.5 \\
\hline Left MCA & $54(54)$ & & 0.79 & 0.056 & 0.34 to 1.79 \\
\hline HTN & $81(72.3)$ & & 0.88 & 0.766 & 0.37 to 2.04 \\
\hline DM & $22(19.8)$ & & 0.43 & 0.124 & 0.153 to 1.252 \\
\hline Afib & $40(36)$ & & 0.88 & 0.77 & 0.39 to 1.88 \\
\hline Smoking & $16(17)$ & & 1.41 & 0.53 & 0.46 to 4.32 \\
\hline CAD & $29(26.8)$ & & 1 & 1 & 0.47 to 2.47 \\
\hline $\mathrm{TICl} \geq 2 \mathrm{~b}$ & 97 (86.6) & & 2.07 & 0.010 & 1.18 to 3.62 \\
\hline Intubation & $13(12.4)$ & & 0.45 & 0.2 & 0.13 to 1.59 \\
\hline Intravenous tPA & $46(41)$ & & 1.6 & 0.20 & 0.75 to 3.63 \\
\hline M1 & $70(62)$ & & 1.2 & 0.53 & 0.57 to 2.89 \\
\hline M2 & $9(8)$ & & 1.18 & 0.8 & 0.27 to 5.00 \\
\hline ICA terminus & $21(18.7)$ & & 0.81 & 0.69 & 0.29 to 2.23 \\
\hline Tandem & $21(18.7)$ & & 2.08 & 0.16 & 0.73 to 5.88 \\
\hline Any ICA & $38(33.9)$ & & & & \\
\hline Basilar & $12(10.7)$ & & & & \\
\hline Number of passes & & $2(1-8)$ & 0.88 & 0.35 & 0.68 to 1.14 \\
\hline Intra-arterial tPA & $3(2.6)$ & & & & \\
\hline Stent retriever & $32(28.5)$ & & & & \\
\hline Merci & $6(5.3)$ & & & & \\
\hline Intra-arterial tPA & $3(2.6)$ & & & & \\
\hline Multiple devices & $5(4.4)$ & & & & \\
\hline Hemorrhagic transformation & $31(27)$ & & & & \\
\hline Parenchymal hematoma & $11(9.8)$ & & & & \\
\hline Symptomatic bleed & $7(6.2)$ & & & & \\
\hline ENT & $4(3.5)$ & & & & \\
\hline $\mathrm{mRS} \leq 2$ at 90 days & $52(46.1)$ & & & & \\
\hline mRS 6 & $35(31)$ & & & & \\
\hline Pure MAT & $66(59)$ & & 1.14 & 0.73 & 0.522 to 2.50 \\
\hline $\begin{array}{l}\text { Aspiration catheter } \\
0.4 / 0.5 / 0.6\end{array}$ & & & 0.75 & 0.45 & 0.36 to 1.55 \\
\hline \multirow[t]{3}{*}{ Aspiration catheter brand } & Navien (84) & & 1.2 & 0.46 & 0.73 to 1.97 \\
\hline & DAC (4) & & & & \\
\hline & Penumbra (23) & & & & \\
\hline
\end{tabular}

${ }^{*}$ Time to procedure: from onset of symptoms or last seen normal to groin puncture.

†Treatment time: from puncture to recanalization.

ASPECT, Alberta Stroke Program Early CT score; CAD, coronary artery disease; DM, diabetes mellitus; ENT, embolization to a new territory; HTN, hypertension; IA, intra-arterial; ICA, internal carotid artery; MAT, manual aspiration thrombectomy; MCA, middle cerebral artery; mRS, modified Rankin Scale; NIHSS, NIH Stroke Scale; TICI, Thrombolysis in Cerebral Infarction. 
indicate that this technique is feasible as a first step approach in a significant proportion of patients. Previous prospective multicenter studies investigating procedural outcomes with stent retrievers have reported TICI $2 \mathrm{~b} / 3$ recanalization rates of $68.5 \%$ and $67.8 \%$ with Solitaire and Trevo, respectively, translating into favorable clinical outcome rates $(\mathrm{mRS} \leq 2)$ of $36.3 \%$ and $40 \%$, respectively. ${ }^{11} 12$ Our results compare favorably with those obtained with this latest generation of FDA-approved neurothrombectomy devices.

Successful revascularization following large vessel occlusion offers patients the best chance for recovery. The recently published Interventional Management of Stroke III trial demonstrated a high correlation between the degree of revascularization and patient outcomes after stroke: $12.7 \%$ of individuals with TICI scores of 0 had favorable neurologic outcomes while those with TICI scores of $1,2 \mathrm{a}, 2 \mathrm{~b}$ and 3 had rates of $27.6 \%, 34.3 \%, 47.9 \%$ and $71.4 \%$, respectively. ${ }^{13}$ The importance of rapid revascularization in eliciting favorable outcomes was underscored by the findings that each 30 min delay in reperfusion was associated with a $10 \%$ reduction in the likelihood of a favorable outcome. ${ }^{14}$ Thus, the ideal treatment offers a high degree of recanalization in the minimal amount of time.

The MAT technique was first described in the cardiac literature and has been shown to be an effective adjunctive treatment for revascularization of coronary arteries. ${ }^{15} 16$ There is a paucity of data pertaining to the application of MAT in the intracranial circulation. Early case reports, primarily in the posterior circulation, posited MAT as a promising technique owing to the reduced time for recanalization, low hemorrhagic risk and possible prevention of distal clot migration. ${ }^{5817}$ More recent publications have confirmed high recanalization rates and improved outcomes in larger series of patients undergoing MAT in the setting of large vessel intracranial occlusions. In their report involving 22 consecutive patients, Kang et al were able to achieve TICI $2 \mathrm{~b} / 3$ recanalization in $81.9 \%$ of patients and favorable outcomes (90-day $\mathrm{mRS} \leq 2$ ) in $45.5 \%$. $^{7}$ In a multiinstitutional carefully selected series of 37 patients, Turk et al demonstrated successful recanalization with aspiration alone in $75 \%$ of patients. ${ }^{18}$ In the largest series published to date, Jankowitz et al studied the outcomes of 191 patients at a single institution. ${ }^{6}$ Although successful recanalization (TICI $2 \mathrm{~b} / 3$ scores) was achieved in $71 \%$ of patients with a favorable outcome (90-day mRS $\leq 2)$ rate of $54 \%$, MAT was preceded by clot manipulation with the Merci device in the majority of cases. Although primarily used as an anchoring tool to help advance the aspiration catheter through the carotid siphon, the Merci device may have facilitated aspiration by disrupting the clot or by pulling it into the catheter tip.

This prospective registry was designed to overcome this concern. Every attempt was made to rely on pure MAT for recanalization with the largest catheter deemed appropriate, even in cases that were considered unlikely to succeed based on tortuous anatomy or small vessels. The goal was to provide a realistic assessment of aspiration as a stand-alone thrombectomy technique. It should not be surprising that this paradigm was initially successful in only $41 \%$ of patients, with another $18 \%$ requiring multiple catheters, or that the median treatment time was $70 \mathrm{~min}$. The latter is longer than that described in recent reports of manual aspiration in acute stroke. ${ }^{18}$ This finding may be explained by the fact that this is a truly consecutive case series of primary aspiration in which an attempt was made to use MAT as first-line treatment in all patients, even in cases where aspiration catheter access was perceived as difficult based on review of the angiographic runs or on other considerations.
We are not aware of another case series describing a similar approach in the literature, and therefore feel that a comparison of procedural times in our study compared with those described in other studies is not meaningful. Furthermore, with regard to other recent non-aspiration case series, our median time to revascularization of $70 \mathrm{~min}$ is comparable to the $77 \mathrm{~min}$ recently published in the North American Solitaire Stent Retriever Acute Stroke registry that included a similar patient population. ${ }^{19}$

Two other points should be noted. First, since aspiration catheters are significantly less expensive than other devices that are commonly used in mechanical thrombectomy, the use of pure MAT as a first-line modality for clot removal has the potential for significant cost reduction, even with the use of multiple catheters. Second, improved technology will facilitate this technique. The evolution of more supportive wires and microcatheters, along with advances in aspiration catheter technology, will facilitate tracking of large-bore aspiration catheters into the MCA. Improved operator experience will also play a significant part.

The limitations of our study include its lack of a prospective design. In addition, lack of core laboratory adjudication of procedural results sheds some concern about the validity of a comparison between the outcomes reported in this study and those obtained through core laboratory adjudicated trials. The analysis across manufacturers of aspiration catheters is limited by the overwhelming predominance of a single brand and a limited number of patients. Nontheless, our study adds to the growing body of literature supporting the use of MAT as part of a multimodality recanalization strategy.

\section{CONCLUSIONS}

In our experience, employing primary MAT as the first-line technique to perform intracranial thrombectomy is associated with reasonable recanalization rates, good clinical outcomes, equivalent safety profiles and a lower cost compared with other mechanical revascularization devices currently on the market in the USA. Further prospective randomized trials of well-matched populations should provide ultimate evidence of benefit with this intra-arterial approach compared with standard medical therapy or other commonly used intra-arterial mechanical thrombectomy devices.

Contributors All authors contributed to the work presented through study design, manuscript composition and critical review.

Competing interests None.

Ethics approval Ethical approval was obtained from the Institutional Review Board at the University of Pittsburgh Medical Center (IRB\# PR008120394).

Provenance and peer review Not commissioned; externally peer reviewed.

\section{REFERENCES}

1 Smith WS, Sung G, Saver J, et al. Mechanical thrombectomy for acute ischemic stroke: final results of the Multi MERCI trial. Stroke 2008;39:1205-12.

2 Anon. Tissue plasminogen activator for acute ischemic stroke. The National Institute of Neurological Disorders and Stroke rt-PA Stroke Study Group. N Engl J Med 1995;333:1581-7.

3 Vink MA, Patterson MS, van Etten J, et al. A randomized comparison of manual versus mechanical thrombus removal in primary percutaneous coronary intervention in the treatment of ST-segment elevation myocardial infarction (TREAT-MI). Catheter Cardiovasc Interv 2011;78:14-19.

4 Tarsia G, De Michele M, Polosa D, et al. Manual versus nonmanual thrombectomy in primary and rescue percutaneous coronary angioplasty. Heart Vessels 2010;25:275-81.

5 Jankowitz BT, Aleu A, Lin R, et al. Endovascular treatment of basilar artery occlusion by manual aspiration thrombectomy. J Neurointerv Surg 2010; 2:110-14.

6 Jankowitz B, Aghaebrahim A, Zirra A, et al. Manual aspiration thrombectomy: adjunctive endovascular recanalization technique in acute stroke interventions. Stroke 2012;43:1408-11. 
7 Kang DH, Hwang YH, Kim YS, et al. Direct thrombus retrieval using the reperfusion catheter of the penumbra system: forced-suction thrombectomy in acute ischemic stroke. AJNR Am J Neuroradiol 2011;32:283-7.

8 Chapot R, Houdart E, Rogopoulos A, et al. Thromboaspiration in the basilar artery: report of two cases. AJNR Am J Neuroradiol 2002;23:282-4.

9 Higashida RT, Furlan AJ, Roberts $\mathrm{H}$, et al. Trial design and reporting standards for intra-arterial cerebral thrombolysis for acute ischemic stroke. Stroke 2003;34: e109-37.

10 Williams DO, Borer J, Braunwald E, et al. Intravenous recombinant tissue-type plasminogen activator in patients with acute myocardial infarction: a report from the NHLBI thrombolysis in myocardial infarction trial. Circulation 1986;73:338-46.

11 Saver JL, Jahan R, Levy El, et al. Solitaire flow restoration device versus the Merci Retriever in patients with acute ischaemic stroke (SWIFT): a randomised, parallel-group, non-inferiority trial. Lancet 2012;380:1241-9.

12 Nogueira RG, Lutsep HL, Gupta R, et al. Trevo versus Merci retrievers for thrombectomy revascularisation of large vessel occlusions in acute ischaemic stroke (TREVO 2): a randomised trial. Lancet 2012;380:1231-40.

13 Broderick JP, Palesch YY, Demchuk AM, et al. Endovascular therapy after intravenous t-PA versus t-PA alone for stroke. N Engl J Med 2013;368:893-903.
14 Mazighi M, Chaudhry SA, Ribo M, et al. Impact of onset-to-reperfusion time on stroke mortality: a collaborative pooled analysis. Circulation 2013;127:1980-5.

15 Burzotta F, Trani C, Romagnoli E, et al. Manual thrombus-aspiration improves myocardial reperfusion: the randomized evaluation of the effect of mechanical reduction of distal embolization by thrombus-aspiration in primary and rescue angioplasty (REMEDIA) trial. J Am Coll Cardiol 2005;46:371-6.

16 Napodano M, Pasquetto G, Sacca S, et al. Intracoronary thrombectomy improves myocardial reperfusion in patients undergoing direct angioplasty for acute myocardial infarction. J Am Coll Cardiol 2003;42:1395-402.

17 Nedeltchev K, Remonda L, Do DD, et al. Acute stenting and thromboaspiration in basilar artery occlusions due to embolism from the dominating vertebral artery. Neuroradiology 2004;46:686-91.

18 Turk AS, Spiotta A, Frei D, et al. Initial clinical experience with the ADAPT technique: a direct aspiration first pass technique for stroke thrombectomy. J Neurointerv Surg 2014;6:231-7.

19 Zaidat 00, Castonguay AC, Gupta R, et al. North American Solitaire Stent Retriever Acute Stroke registry: post-marketing revascularization and clinical outcome results. J Neurointerv Surg 2014;6:584-8. 